\title{
Balancing of Common DC-Bus Parallel Connected Modular Inductive Power Transfer Systems
}

\author{
Hakan Polat, Enes Ayaz, Ogün Altun, Ozan Keysan
}

\begin{abstract}
The aim of this paper is to design a modular, fault tolerant multi-transmitter(Tx)/multi-receiver(Rx) parallel connected common DC bus inductive power transfer(IPT) system to replace slip rings in wind turbines or brushless exciters. In parallel connected common DC bus systems, current unbalance is a major issue that results in thermal stresses and over current or voltages. In this paper, two different new current balancing methods are proposed: Cross-coupled $R x$ modules and intentional de-tuning of $R x$ side resonant frequency. These methods are investigated both analytically and experimentally for a single Tx and two Rx system for a $500 \mathrm{~W}$ prototype. The proposed methods are tested independently, and then the combined current balancing method is also investigated. For the same misalignment case, cross-coupled/de-tuned has a $55.8 \%$ current balancing improvement compared to de-coupled/fully-tuned topology.
\end{abstract}

Index Terms-Wireless power transfer, inductive power transfer, modular design, common DC bus, current balancing

\section{LIST OF ABBREVIATIONS}

$\alpha \quad 2^{\text {nd }} \mathrm{Rx}$ current phase

$\beta \quad 1^{\text {st }} \mathrm{Rx}$ current phase

$\delta \quad$ Angle between Rx currents

$\omega \quad$ Operating angular frequency

$\omega_{0} \quad$ Resonant angular frequency

$\omega_{R x} \quad$ Resonant angular frequency of Rx side resonant tank

$\omega_{T x} \quad$ Resonant angular frequency of Tx side resonant tank

$\theta \quad$ Tx current phase

$C_{R x} \quad \mathrm{Rx}$ side resonant capacitor

$C_{T x} \quad$ Tx side resonant capacitor

$D_{\text {in }} \quad$ Inner diameter of an IPT coil

$D_{\text {out }} \quad$ Outer diameter of an IPT coil

$f_{0} \quad$ Resonant frequency

$I_{R x, n} \quad n^{\text {th }} \mathrm{Rx}$ side AC input current

$I_{T x} \quad$ Tx side AC input current

$L_{R x, n} n^{t h} \mathrm{Rx}$ inductance

$L_{T x, n} \quad n^{t h}$ Tx inductance

$M \quad$ Mutual inductance

$M_{1} \quad$ Mutual inductance between Tx and the first Rx

$M_{2} \quad$ Mutual inductance between Tx and the second Rx

$M_{s} \quad$ Mutual inductance between $\mathrm{Rx}$ coils

$R_{L} \quad$ Load Resistance

$R_{e q u, n}$ Reflected load resistance seen by the $n^{t h} \mathrm{Rx}$

$V_{i n, D C}$ DC input voltage

(C) 2021 IEEE. Personal use of this material is permitted. Permission from IEEE must be obtained for all other uses, in any current or future media, including reprinting/republishing this material for advertising or promotional purposes, creating new collective works, for resale or redistribution to servers or lists, or reuse of any copyrighted component of this work in other works.

The article has been accepted for publication by IEEE DOI:10.1109/JESTPE.2021.3074888.

Corresponding Author: Ozan Keysan, keysan@metu.edu.tr
$V_{\text {in }} \quad$ Fundamental component of square input voltage

$V_{\text {out }, D C}$ DC output voltage

$V_{\text {out }} \quad$ Voltage seen across reflected load resistance

$V_{R x, n} \quad$ Induced voltage on the $n^{t h} \mathrm{Rx}$ coil

$Z_{r} \quad$ Reflected impedance seen across the Tx side inductor

$Z_{T x} \quad$ Reflected impedance seen across the Tx side

\section{INTRODUCTION}

Inductive power transfer (IPT) systems have become more popular in recent years [1]. They are used in a wide range from low power applications to high power applications [2]. Cordless design and spatial flexibility of IPTs led them to be used in applications such as portable chargers [3], [4], biomedical implements [5], electric cars [6], etc. They also provide electrical safety and galvanic isolation. IPT systems also allow the use of multi-Tx, and multi-Rx [7]-[12]. Multi-Tx coils are implemented to obtain higher power ratings and to achieve modularity where power rating can be adjusted according to demand [13]. Multi-Rx coils can also be used to achieve higher power levels with lower semiconductor ratings. $\mathrm{Rx}$ modules can be connected in parallel or series or each module may supply independent loads [14]. Power sharing between parallel Rx's, also known as load balancing, is challenging due to variation of $\mathrm{Tx}-\mathrm{Rx}$ couplings. This problem can be either solved by replacing passive rectifiers with controlled rectifiers at the $\mathrm{Rx}$ side [4], [15] or by using an additional DC-DC converter [16]. Although these solutions are viable for compensating large coupling differences between parallel connected Rx modules, they are more complex and require additional components, which increases the size and the cost.

In the literature, multiple coil configurations are also used to increase miss-alignment tolerances. However, power unbalance is still a major issue. In [17], series-series and series-parallel compensation methods are investigated for parallel common DC-bus connected systems. In seriesseries compensation, de-tuning is a viable option to reduce unbalances, but it cannot be used for the series-parallel case. In [18], two Rx coils are placed orthogonally such that the miss-alignment problem is reduced for angular displacements. Again series-parallel topology is used and connected to a common DC bus, with a current smoothing inductor.

In this paper, two current balancing methods for seriesseries compensation are presented; cross-coupling between the Rx modules and intentional de-tuning of Rx side resonant frequency. The proposed methods increase efficiency, decrease 
thermal stress on the semiconductors without the need for active rectifiers or extra converters.

In the first section, the structure and parameters of the 1Tx$2 \mathrm{Rx}$ system will be introduced. Then, the current unbalance problem will be described briefly and confirmed by simulations. In the next sections, the effect of cross-coupling and intentional de-tuning of the $\mathrm{Rx}$ side on the current sharing will be discussed. Proposed methods will be later proven experimentally.

\section{System Structure and Problem Definition}

The aim is to design a contactless slip-ring that can be mounted directly around a rotating shaft. Since the Rx side rotates with the shaft, the airgap clearance stays constant during operation; however, changes in the magnetic coupling between Tx-Rx modules are inevitable. Therefore, a seriesseries topology is selected since the resonant frequency is independent of coupling and electrical loading. To further increase the reliability, a modular structure is selected. This way, during a single Rx module open circuit fault, the power transfer without over-loading the Tx side is possible. Having modularity increases the fault tolerance as well as reduces the semiconductor stresses. Moreover, easy mount and dismounting can be made. Compared to a single archimedean spiral IPT coil, a unique coil geometry is introduced. With the proposed IPT coil geometry, the eddy loss on the rotor shaft is reduced. The efficiency of a single Tx coil will be slightly higher than the proposed coil geometry due to extra end windings. However, the increase in the fault tolerance, modularity and mechanical aspects are the main advantages.

The proposed system (as presentedin Fig. 1-a) consists of two Tx side and four Rx side modules. Although the magnetic coupling between the Tx-Rx modules are varying with rotation, the reflected impedances on the $\mathrm{Tx}$ sides are equal since the system is symmetric. Therefore, for all rotational angles magnetic couplings are equal. In Fig. 1-b, the variation of magnetic couplings between the $\mathrm{Tx}$ and $\mathrm{Rx}$ coils with rotation are presented. In Fig. 1-c, variation of coupling between a single $\mathrm{Rx}$ and a $\mathrm{Tx}$ modules are presented. Since the Tx currents are the same, the induced voltage on the $\mathrm{Rx}$ coil is directly related to the sum of mutual inductances where any difference, which is denoted by $\Delta M$, results in maximum current unbalances. In this paper, the position where $\Delta M$ is maximum, is denoted as the miss-aligned position, the position where $\Delta M$ is minimum is denoted as the aligned position.

Although the purpose is to design a rotational $2 \mathrm{Tx}-4 \mathrm{Rx}$ system with a common DC bus, the current balancing analysis can be performed for $1 \mathrm{Tx}-2 \mathrm{Rx}$ system structure due to symmetry. Tx modules having equal currents can be combined, and any adjacent Rx modules having different magnetic couplings can be used. In Fig. 2, 1Tx-2Rx structure is presented. A fullbridge converter is used on the Tx side, and full-bridge passive diode rectifiers are used on the Rx coils connected to a single load.

\section{A. Problem Definition}

In parallel-connected Rx modules, maintaining equal current sharing between the modules is challenging as the module with the higher magnetic coupling becomes the main power delivery path. The output voltage is set by the module with higher coupling, and the rectifier diodes on the other module are blocked, which results in unbalanced current distribution as shown in Fig. 3. At this stage, no current balancing methods are introduced. The operating frequency is set to a frequency slightly higher than the resonant frequency to ensure an operation at the inductive region, which guarantees zero voltage switching at $\mathrm{Tx}$ side switches. The unbalanced case has $10 \%$ mutual inductance difference and it corresponds to misaligned (M) points in Fig 1-c.

\section{B. Rectifier Effect and Load Sharing}

In the previous section, the main phenomenon behind the unequal current sharing between Rx modules was explained. However, parallel-connected Rx modules with a common DC bus (see Fig. 2) requires further analysis. Although common DC voltage bus equalizes the voltage output of each $\mathrm{Rx}$ module, it doesn't mean equal power sharing. Actually, the reflected load resistance varies due to coupling unbalance between Tx and Rx modules. Since the system operates close to the design resonant frequency, the impedance of series connected LC resonant tank can be assumed to be zero. Then, it is possible to say that induced voltages at $\mathrm{Rx}$ modules can be conceivable as output voltage as shown in (1) and (2). This is also valid for Tx, and the equality is shown in (3).

$$
\begin{gathered}
V_{R x, 1}=j \omega M_{1} I_{T x} \\
V_{R x, 2}=j \omega M_{2} I_{T x} \\
V_{i n}=-j \omega M_{1} I_{R x, 1}-j \omega M_{2} I_{R x, 2}
\end{gathered}
$$

First, let us consider the equal coupling case without any cross-coupling. The phasor diagram of the input and induced voltages at Rx's are shown Fig. 4. In this case, the induced voltage of both $\mathrm{Rx}$ coils is equal to the output voltage. Thus, the designed circuit operates as desired, and Rx's share the power in equal. In the case of coupling mismatch, the Rx module with higher coupling has a higher induced voltage on its Rx side IPT coil, which is later rectified using the diode rectifier. The passive rectifier diodes on the less coupled module are reverse-biased, and hence most of the power is transferred by a single module. Due to the current unbalance, the reflected load resistance $R_{e q}$ is lower for the higher coupled module. For parallel connected common DC-bus systems, the voltage at the input of the diode rectifier at the $\mathrm{Rx}$ side is equal. Therefore, a lower module current indicates a higher $R_{e q}$. For each parallel-connected $\mathrm{Rx}$ module, there exists another $R_{e q}$ value that satisfies (4), where $n$ is the total number of parallelconnected modules.

$$
R_{e q, 1}\left\|R_{e q, 2}\right\|(\ldots) \| R_{e q, n}=\frac{8}{\pi^{2}} R_{L}=R_{r e f}
$$

However, $R_{e q}$ equals to $R_{r e f} / n$ when the module currents are balanced. For unbalanced current distribution, this value 


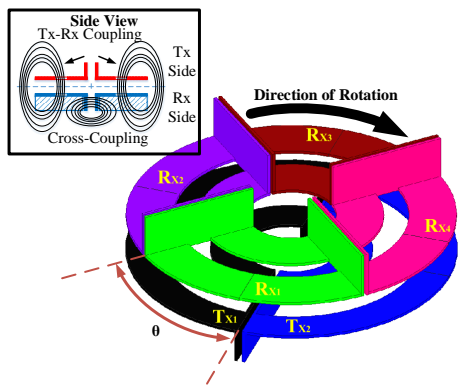

(a) System structure.

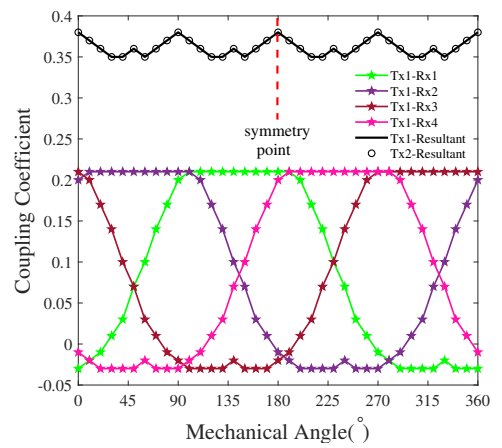

(b) Coupling of a transmitter with respect to full rotation

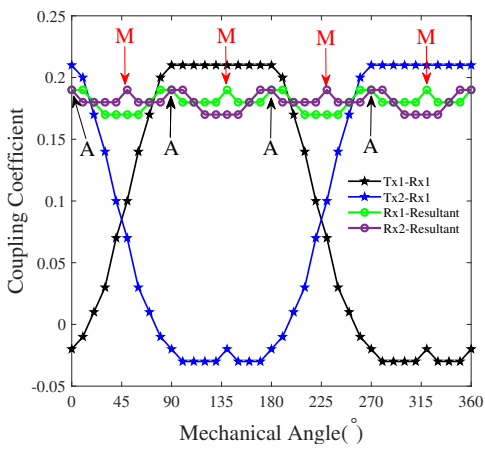

(c) Coupling of two receivers with respect to full rotation

Fig. 1. The system structure and change in couplings for a full rotation. "M" stands for maximum misalignment and "A" stands for fully aligned case.

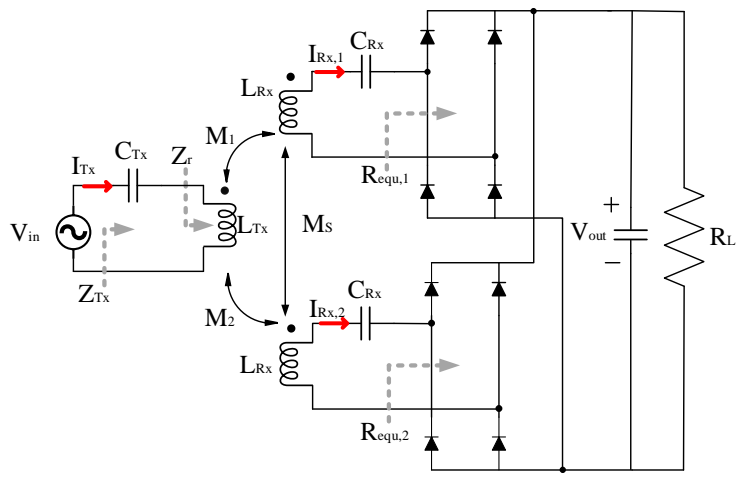

Fig. 2. Parallel connected Rx's with common DC bus.

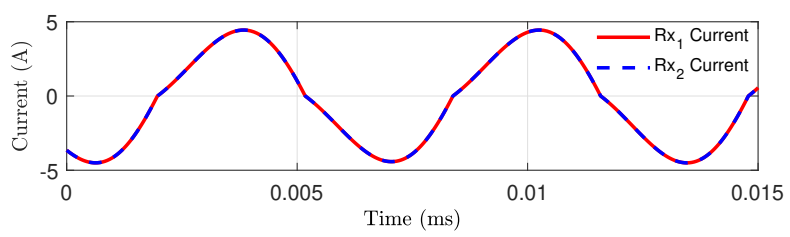

(a) $150 \mathrm{kHz}$ Decoupled Receivers Fully-Aligned Condition

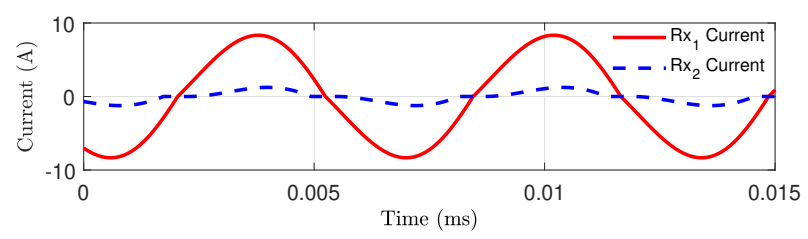

(b) $150 \mathrm{kHz}$ Decoupled Receivers Misaligned Condition

Fig. 3. The change in the receiver currents for equal and different magnetic coupling.

depends on operation frequency, difference in the mutual inductances, Tx side currents $I_{T x, n}$.

\section{Current Balancing Methods}

The current balancing in parallel connected common DCbus systems is necessary to achieve uniform power sharing. The current balancing effect of addition of cross-coupling

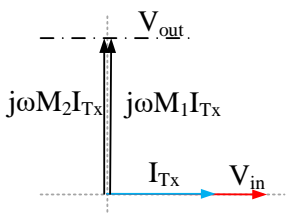

(a) $\mathrm{M}_{1}=\mathrm{M}_{2}$

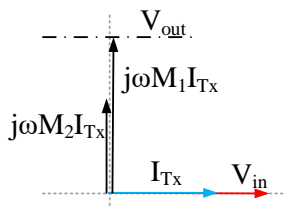

(b) $\mathrm{M}_{1} \neq \mathrm{M}_{2}$
Fig. 4. Rx side induced voltages for equal and unequal magnetic couplings.

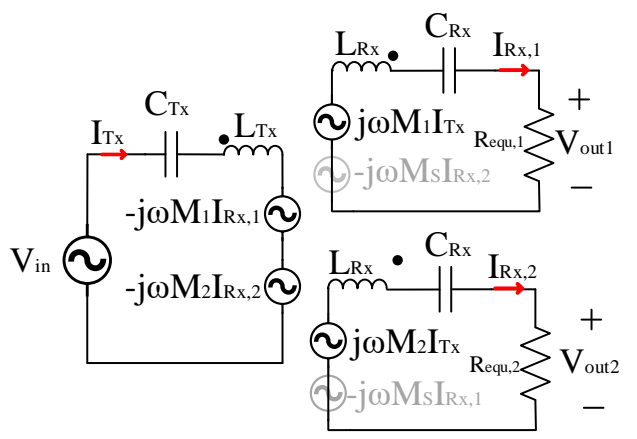

Fig. 5. Parallel Rx modules with and without cross-coupling.(Grayports show the cross-coupling components).

between Rx coils and detuning the Rx resonant frequency will be investigated.

\section{A. Effect Of Receiver Cross-Coupling}

The first proposed method for current balancing is to introduce cross-coupling between the Rx modules. Then the first harmonic approximation (FHA) circuit representation of the system can be represented as in Fig. 5.Compared to the case with no cross-coupling, there exist additional induced voltage components in the FHA circuit. The receiver with the larger coupling is named as the dominant $\mathrm{Rx}$ module, and the other is named as recessive Rx module. It is possible to observe the effect of cross-coupling using a phasor diagram, as shown in Fig. 6. In case of an unbalance between the module currents, the dominant module induces EMF on the 
recessive Rx coil, which lags the dominant module current by $90^{\circ}$ as seen in Fig. 4. The vectorial sum of the induced EMF components on the recessive module due to Tx and dominant $\mathrm{Rx}$ module becomes higher. Thus, the output voltages of $\mathrm{Rx}$ modules are equalized, and power unbalance between receivers is avoided by this cross-coupling component.

In Fig. 7, the effect of cross-coupling on the receiver currents is given. The improvement was given in Fig. 3. Receiver currents are equal in the ideal case with cross-coupled, as shown in Fig. 7-a. However, if the mutual inductances between Tx and Rx modules are not identical but comparable, the majority of the power flows from a single module. In Fig. 7-b, one of the receiver mutual inductance between the transmitter is $10 \%$ smaller than the other and the cross-coupling is introduced as $70 \%$ of mutual inductance between receiver and transmitter. It can be observed that the current/power distribution is significantly improved by this cross-coupling component.

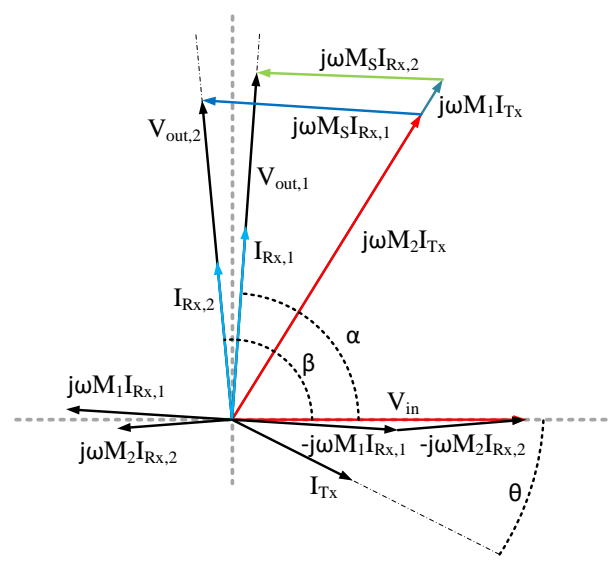

Fig. 6. Phasor diagram for common DC bus parallel connected Rx modules with cross-coupling. The circuit components was given in Fig. 2.

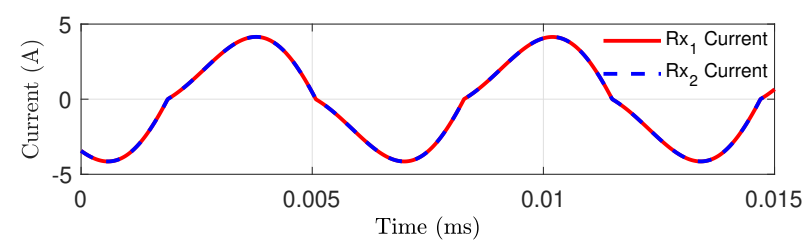

(a) $150 \mathrm{kHz}$ Cross-coupled Fully-Aligned Condition

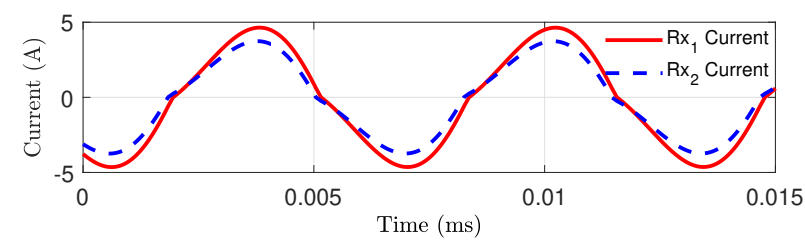

(b) $150 \mathrm{kHz}$ Cross-coupled Misaligned Condition

Fig. 7. Effect of cross-coupling on the Rx side current sharing.

An analytical model is developed to determine the required optimum value of the cross-coupling. Although it may seem beneficial to increase the cross-coupling as much as possible, it is not feasible as increasing the coil winding length increases the resistive losses. In accordance with Fig. 5, the input and output voltages are defined as phasors as in Fig. 6 for the $1 T x-2 R x$ system with a resonant frequency of $f_{0}$.

$$
\begin{gathered}
V_{\text {out }, 1}=j \omega M_{1} I_{T x}+j \omega M_{s} I_{R x, 2} \\
V_{\text {out }, 2}=j \omega M_{2} I_{T x}+j \omega M_{s} I_{R x, 1} \\
V_{\text {in }}=-j \omega M_{1} I_{R x, 1}-j \omega M_{2} I_{R x, 2}
\end{gathered}
$$

The magnitudes of the output voltages are equal but the phases depend on current of each Rx modules. The equations $(5,6,7)$ in the phasor domain are transferred to time-domain equations $(8,9,10)$ by considering the parameters shown in Fig. 6 .

$$
\begin{array}{r}
V_{\text {out }} \sin (\omega t-\alpha)=\omega M_{1} I_{T x} \cos (\omega t-\theta) \\
+\omega M_{s} I_{R x, 2} \cos (\omega t-\beta) \\
V_{\text {out }} \sin (\omega t-\beta)=\omega M_{2} I_{T x} \cos (\omega t-\theta) \\
+\omega M_{s} I_{R x, 1} \cos (\omega t-\alpha) \\
V_{\text {in }} \sin (\omega t)=-\omega M_{1} I_{R x, 1} \sin (\omega t-\alpha) \\
-\omega M_{2} I_{R x, 2} \sin (\omega t-\beta)
\end{array}
$$

After the conversion, the orthogonal set can be divided into two parts as follows:

$$
\begin{gathered}
V_{\text {out }} \sin (\alpha)=-\omega M_{1} I_{T x} \cos (\theta)-\omega M_{s} I_{R x, 2} \cos (\beta) \\
V_{\text {out }} \cos (\alpha)=\omega M_{1} I_{T x} \sin (\theta)-\omega M_{s} I_{R x, 2} \sin (\beta) \\
V_{\text {out }} \sin (\beta)=-\omega M_{2} I_{T x} \cos (\theta)-\omega M_{s} I_{R x, 1} \cos (\alpha) \\
V_{\text {out }} \cos (\beta)=\omega M_{2} I_{T x} \sin (\theta)-\omega M_{s} I_{R x, 1} \sin (\alpha) \\
0=-\omega M_{1} I_{R x, 1} \cos (\alpha)-\omega M_{2} I_{R x, 2} \cos (\beta) \\
V_{\text {in }}=-\omega M_{1} I_{R x, 1} \sin (\alpha)-\omega M_{2} I_{R x, 2} \sin (\beta)
\end{gathered}
$$

The system parameters are shown in Table I for a 1Tx$2 \mathrm{Rx}$ system. By considering the input parameters, the output voltage is calculated in (17) and the projection of the Tx current on the Tx voltages is calculated (18) by neglecting the losses for now.

$$
\begin{gathered}
V_{\text {out }}=\left(I_{R x, 1}+I_{R x, 2}\right) R_{L} \\
I_{p f}=I_{p}(\theta)=\frac{V_{\text {out }}\left(I_{R x, 1}+I_{R x, 2}\right)}{V_{\text {in }}}
\end{gathered}
$$

For negative cross-coupling, it is known that the dominant $\mathrm{Rx}$ current leads the input voltage in the range of 0 and 90 degrees. Also, it is known that the recessive Rx current leads the input voltage in the range of 90 and 180 degrees. The angles of Rx currents with respect to the input voltage can be found by using (15) and (16).

$$
\begin{gathered}
\delta=180-\arccos \left(\frac{V_{i n}^{2}-\omega^{2} M_{1}^{2} I_{R x, 1}^{2}-\omega^{2} M_{2}^{2} I_{R x, 1}^{2}}{-2 \omega^{2} M_{1} M_{2} I_{R x, 1}^{2}}\right) \\
\alpha=-\arctan \left(\frac{\omega M_{1} I_{s 1}+\omega M_{2} I_{R x, 1} \cos (\delta)}{\omega M_{2} I_{R x, 1} \sin (\delta)}\right) \\
\beta=\alpha-\delta
\end{gathered}
$$


It is possible to find the required cross-coupling $\left(\mathrm{M}_{\mathrm{S}}\right)$ by using (11) and (12) or (13) and (14). In these equations, $\theta$ and $M_{s}$ are unknown parameters. These equations can be reshaped in the linear form by changing the parameters as $\tan (\theta)$ and $M_{s}$. The equations are brought $y=A^{-1} B$ form where $\mathrm{A}, \mathrm{B}$ and $\mathrm{y}$ are shown in (22), (23) and (24).

$$
\begin{gathered}
A=\left[\begin{array}{cc}
\omega M_{2} I_{p f} & \omega I_{R x, 1} \cos (90+\alpha) \\
0 & \omega I_{R x, 1} \sin (90+\alpha)
\end{array}\right] \\
B=\left[\begin{array}{c}
V_{\text {out }} \sin (\beta) \\
V_{\text {out }} \sin (\beta)-\omega M_{2} I_{p f}
\end{array}\right] \\
y=\left[\begin{array}{c}
\tan \theta \\
M_{s}
\end{array}\right]
\end{gathered}
$$

The required cross coupling between Rx modules for a desired unbalance constraint can be calculated as shown in (25) which depends on the input parameters given in Table I. Although, the analitically deriven $M_{s}$ seems to depend on $M_{2}$ only, $I_{p f}$ and $\mathrm{I}_{\mathrm{Rx}, 1}$ depend on the difference in the mutual inductances.

$$
M_{s}=\frac{V_{\text {out }} \sin (\beta)-\omega M_{2} I_{p f}}{\omega I_{R x, 1} \cos (90+\alpha)}
$$

The scenario of a 5\% difference between mutual inductances is presented in Table I. For this scenario, the desired current balancing ratio (CBR) is chosen as82\% (defined in (44)), and the required cross-coupling is calculated as 0.167 $\left(\mathrm{M}_{\mathrm{S}}=11.02 \mu \mathrm{H}\right)$. The simulation results for the derived values are given in Fig. 8.

TABLE I

CROSS-COUPLED ANALYTICAL INPUT PARAMETERS AND RESULTS

\begin{tabular}{ll|ll}
\multicolumn{1}{c}{ Input Parameters } & Values & Derived Parameters & Values \\
\hline $\mathrm{f}_{0}$ & $150 \mathrm{kHz}$ & $\mathrm{V}_{\text {out }}(\mathrm{rms})$ & $112.2 \mathrm{~V}$ \\
$\mathrm{~L}_{T x}$ & $82 \mu H$ & $\alpha$ & $76.7^{\circ}$ \\
$\mathrm{L}_{R x}$ & $66 \mu H$ & $\beta$ & $110.8^{\circ}$ \\
$\mathrm{M}_{1}$ & $14.71 \mu H$ & $\mathrm{I}_{T x}(r m s)$ & $8.6 \mathrm{~A}$ \\
$\mathrm{M}_{2}$ & $13.97 \mu H$ & $\theta$ & $19.5^{\circ}$ \\
$\mathrm{V}_{i n}(r m s)$ & $90 \mathrm{~V}$ & $\mathrm{M}_{s}$ & $11.02 \mu H$ \\
$\mathrm{I}_{R x, 1}(\mathrm{rms})$ & $4.1 \mathrm{~A}$ & & \\
$\mathrm{I}_{R x, 2}(\mathrm{rms})$ & $2.8 \mathrm{~A}$ & & \\
$\mathrm{R}_{L}$ & $20 \Omega$ & & \\
\hline
\end{tabular}

\section{B. Intentional De-tuning of the Receiver Side Resonant Tank}

It is observed that increasing operating frequency (while keeping the resonant frequency constant) results in a more balanced current distribution. However, as the operating frequency deviates further from the resonant frequency, the output voltage decreases, and hence the power output reduces. The same current balancing effect can be achieved by intentional de-tuning of the receiver resonant tank for unbalanced modules. Feng et al. investigate a detuned 1Tx-1Rx system, and the effect of varying coupling coefficient is analyzed [19]. In this section, the effect of Rx side detuning on current sharing is expanded for a $1 T x-2 R x$ system. Firstly, an analytical model is derived for a single Tx and single Rx system, and the effect of operation frequency and resonant tank frequencies

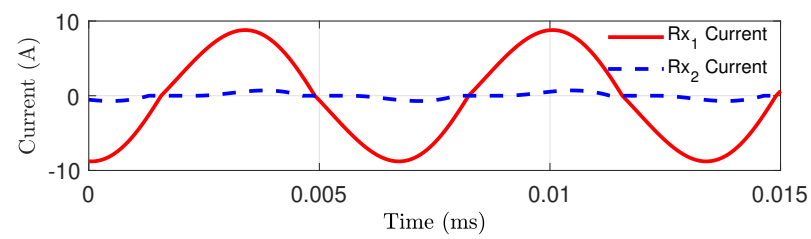

(a) $150 \mathrm{kHz}$ Decoupled Condition $\left(\mathrm{I}_{\mathrm{Rx} 1}=6.2 \mathrm{~A}_{\mathrm{rms}}, \mathrm{I}_{\mathrm{Rx} 2}=0.5 \mathrm{~A}_{\mathrm{rms}}\right)$

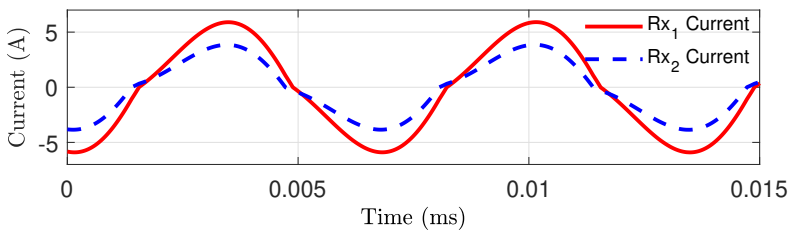

(b) $150 \mathrm{kHz}$ Cross-Coupled Condition $\left(\mathrm{I}_{\mathrm{Rx} 1}=4.1 \mathrm{~A}_{\mathrm{rms}}, \mathrm{I}_{\mathrm{Rx} 2}=2.7 \mathrm{~A}_{\mathrm{rms}}\right)$

Fig. 8. Effect of cross-coupling on the current sharing for missaligned case.

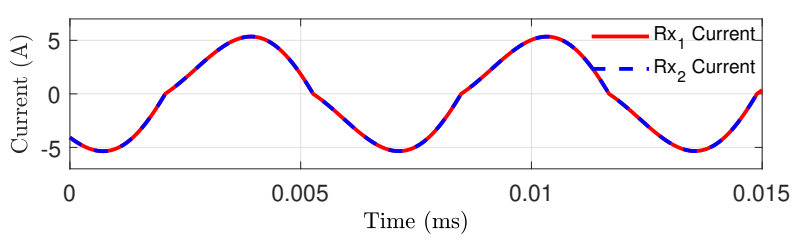

(a) $135 \mathrm{kHz}$ Decoupled Receivers Fully-Aligned Condition

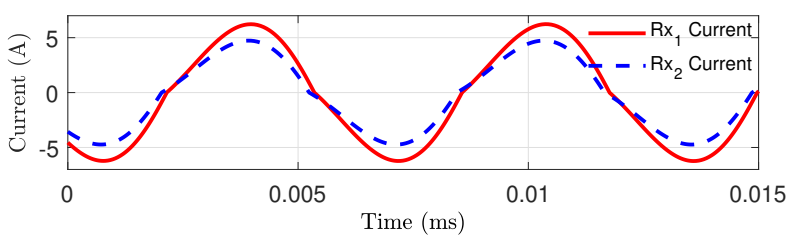

(b) $135 \mathrm{kHz}$ Decoupled Receivers Misaligned Condition

Fig. 9. Effect of detuning on the current sharing.

are discussed. Secondly, a similar approach is used to analyze the effect of Rx side de-tuning for a 1Tx-2Rx system.

The FHA circuit representation of the 1Tx-1Rx system is presented in Fig. 10. The Tx and Rx resonant tank impedances are presented in (26) and (27). Then, the impedance matrix (28) can be solved for the transmitter and receiver currents as shown in (29) and (30).

$$
\begin{gathered}
j L_{T x}\left(\frac{\omega^{2}-\omega_{T x}^{2}}{\omega^{2}}\right)=j L_{T x}\left(\Delta \omega_{T x}\right) \\
j L_{R x}\left(\frac{\omega^{2}-\omega_{R x}^{2}}{\omega^{2}}\right)=j L_{R x}\left(\Delta \omega_{R x}\right) \\
{\left[\begin{array}{c}
V_{i n} \\
0
\end{array}\right]=\left[\begin{array}{cc}
Z_{T x} & j \omega M \\
j \omega M & -Z_{R x}
\end{array}\right]\left[\begin{array}{c}
I_{T x} \\
I_{R x}
\end{array}\right]} \\
I_{p}=\frac{V_{i n}\left(-L_{R x} \Delta \omega_{R x}+j R_{L}\right)}{L_{T x} R_{L} \Delta \omega_{T x}+j\left(M^{2} \omega^{2}+L_{T x} L_{R x} \Delta \omega_{T x} \Delta \omega_{R x}\right)} \\
I_{s}=\frac{M_{i n}}{L_{T x} R_{L} \Delta \omega_{T x} \Delta \omega_{R x}+j\left(M^{2} \omega^{2}+L_{T x} R_{L} \Delta \omega_{T x}\right)}
\end{gathered}
$$

In conventional series-series IPT systems, the Tx and Rx side resonant tank frequencies are chosen equally in order to achieve load/coupling independent operation. However, it 


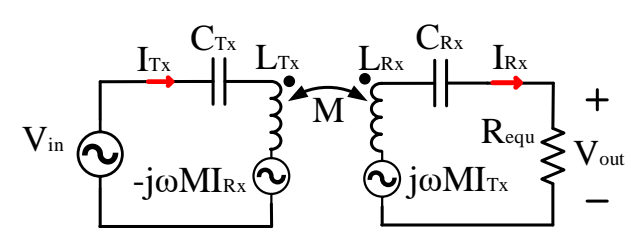

Fig. 10. First harmonic approximation of single transmitter single receiver IPT system.

is shown in this paper that for common DC bus parallelconnected multi-Rx systems, the current sharing can be improved by intentional de-tuning. When both Tx and Rx frequencies are equal to each other, the Tx and Rx currents can be derived as in (31) and (32) respectively. Under this condition, the Rx current lags the Tx current by $90^{\circ}$.

$$
\begin{gathered}
I_{T x}=\frac{V_{i n} R_{L}}{M^{2} \omega^{2}} \\
I_{R x}=\frac{-j V_{i n}}{M \omega}
\end{gathered}
$$

However, if the Rx resonant tank is de-tuned and the operating frequency is kept at the resonant frequency of the Tx, the Tx and Rx currents can be derived as in (33) and (34).

$$
\begin{gathered}
I_{T x}=\frac{V_{i n} R_{L}}{M^{2} \omega^{2}}+\frac{j V_{i n} L_{R x} \Delta \omega_{R x}}{M^{2} \omega^{2}} \\
I_{R x}=\frac{-j V_{i n}}{M \omega}
\end{gathered}
$$

Therefore, detuning the Rx side results in either leading or lagging Tx current while the Rx current stays the same. If the resonant frequencies of the Rx's are chosen below the resonant frequency of the Tx side, the overall system resonant frequency will be above the resonant frequency of the transmitter and vice-versa. The analytical derivations can be expanded for a 1 Tx-2Rx system as shown in Fig. 5. The cross-coupling is ignored during derivation of $\mathrm{Rx}$ side detuning, and the rectifier can be modeled as a variable load with common output voltage. The matrix equations for the system are shown in (35) and the receiver currents as in (36) and (37) are obtained.

$$
\begin{gathered}
{\left[\begin{array}{c}
V_{i n} \\
0 \\
0
\end{array}\right]=\left[\begin{array}{ccc}
Z_{T x} & j \omega M_{1} & j \omega M_{2} \\
j \omega M_{1} & -Z_{R x, 1} & 0 \\
j \omega M_{1} & 0 & -Z_{R x, 2}
\end{array}\right]\left[\begin{array}{c}
I_{T x} \\
I_{R x, 1} \\
I_{R x, 2}
\end{array}\right]} \\
I_{R x, 1}=\frac{j I_{T x} M_{1} \omega^{2}}{R_{L 1} \omega+j\left(L_{R x}^{2}\left(\omega^{2}-\omega_{R x}^{2}\right)\right)} \\
I_{R x, 2}=\frac{j I_{T x} M_{2} \omega^{2}}{R_{L 2} \omega+j\left(L_{R x}^{2}\left(\omega^{2}-\omega_{R x}^{2}\right)\right)}
\end{gathered}
$$

Although the load distribution of the modules are unknown, the magnitude of the voltages are equal as shown in (38). The extended formulation is shown in (39).

$$
R_{L 1}\left|I_{R x, 1}\right|=R_{L 2}\left|I_{R x, 2}\right|
$$

$$
\begin{array}{r}
I_{T x} M_{1} \omega^{2} R_{L 1} \sqrt{R_{L 1}^{2} \omega^{2}+L_{R x}^{4}\left(\omega^{2}-\omega_{R x}^{2}\right)^{2}} \\
-I_{T x} M_{2} \omega^{2} R_{L 2} \sqrt{R_{L 2}^{2} \omega^{2}+L_{R x}^{4}\left(\omega^{2}-\omega_{R x}^{2}\right)^{2}}=0
\end{array}
$$

When $\omega=\omega_{R x}$, (40) can be derived. For this equation to be valid, either $R_{L 1}$ or $R_{L 2}$ must go to infinity since $M_{1} \neq M_{2}$.

$$
I_{T x} M_{1} \omega^{3} R_{L 1} R_{L 2}-I_{T x} M_{2} \omega^{3} R_{L 1} R_{L 2}=0
$$

However, if $\omega \neq \omega_{s}$ and with the assumption of a high quality factor, (41) can be obtained. Using the same assumptions, (39) can be written as in (42).

$$
\begin{aligned}
& \sqrt{R_{L 2}^{2} \omega^{2}+L_{R x}^{4}\left(\omega^{2}-\omega_{R x}^{2}\right)^{2}}=L_{R x}^{2}\left(\omega^{2}-\omega_{R x}^{2}\right) \\
& I_{T x} \omega^{3} L_{R x}^{2}\left(\omega^{2}-\omega_{R x}^{2}\right)\left(M_{1} R_{L 1}-M_{2} R_{L 2}\right)=0
\end{aligned}
$$

By simplifying (42) and under the assumption that the Rx side is detuned and the quality factor is high, the load distribution is inversely proportional to the mutual inductances ratio as shown in (43).

$$
\frac{R_{L 1}}{R_{L 2}}=\frac{M_{2}}{M_{1}}
$$

In summary, increasing operation frequency while keeping the same resonant tank at Tx and Rx results in a better current distribution. However, as the frequency gets further into the inductive region, the power output decreases. If the $\mathrm{Rx}$ side resonant frequency is decreased, the maximum balancing can be achieved as provided in (43). Moreover, with detuning operation at the resonant frequency is still achievable without any loss in the maximum power delivery capability. Again without any change, frequency control can be used to control the power output. The simulation result for the normal and de-tuned receiver is given in Fig. 9.

A major disadvantage of a detuned Rx side is the variation of the resonant frequency for different loading and couplings. In the literature, a series-series topology is usually selected for its independence of resonant frequency for variable operating conditions [6], [20]. In our final $2 \mathrm{Tx}-4 \mathrm{Rx}$ design, the variation of the reflected impedance is minimized. As the $\mathrm{Rx}$ modules rotate, the Tx side the magnetic coupling with another $\mathrm{Rx}$ module while it decreases with the other $\mathrm{Rx}$ module. Therefore, change in the resonant frequency is minimal in our system.

\section{EXPERIMENTAL RESUlts}

The $1 T x-2 R x$ system is presented in Fig. 11, where there is a single full-bridge converter, and the other Rx circuits consist of passive diode full-bridge rectifiers connected in parallel to the same load.

For the cross-coupled $\mathrm{Rx}$ modules, the coupling is set to be $k_{c}=-0.15$, and for the detuned $\mathrm{Rx}$ side, the resonant frequency of the Rx side is adjusted to $135 \mathrm{kHz}$. For 1Tx$2 \mathrm{Rx}$ experiments, a half-circular structure is used where a slight misalignment between a single $\mathrm{Tx}$ and two adjacent $\mathrm{Rx}$ modules is given where the maximum $\Delta M$ is satisfied, which was previously presented in Fig. 1. For 1Tx-2Rx experiments, the output power is set to $500 \mathrm{~W}$, and similarly, for the 


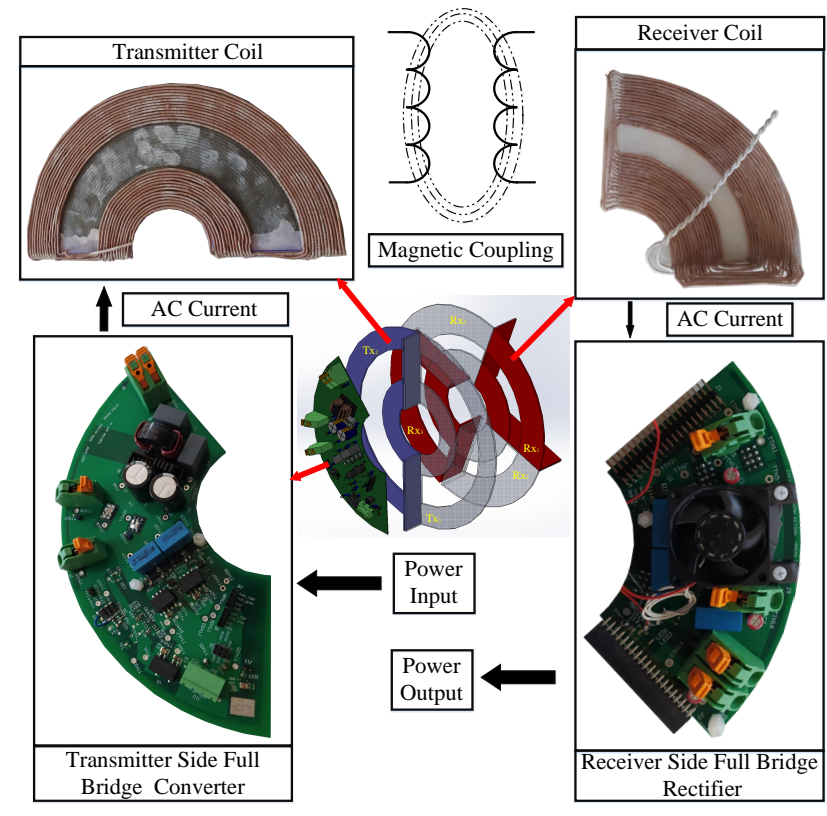

Fig. 11. Test setup illustration.

$2 \mathrm{Tx}-4 \mathrm{Rx}$ case, this number is doubled to $1000 \mathrm{~W}$. For all experiments, the $R_{L}$ is kept constant. Therefore, keeping the output power constant also means a constant output voltage $V_{\text {out }, D C}$. The experimental setup parameters are listed in Table II.

TABLE II

EXPERIMENTAL SETUP PARAMETERS

\begin{tabular}{ll}
\hline & \\
Tx side MOSFET & BSC600N25NS3G \\
Tx side Gate Driver & 2EDF7275F \\
Rx side Recrifier Diodes & C3D10060G \\
Litz Wire & 400x0.08mm $\left(2 \mathrm{~mm}^{2}\right)$ \\
Tx coil $\mathrm{D}_{\text {in }}$ and $\mathrm{D}_{\text {out }}(\mathrm{mm})$ & $70-280$ \\
Tx coil \# of turns & 13 \\
Tx coil inductances $(\mu \mathrm{H})$ & $83.1-83.5$ \\
$\mathrm{Rx}$ coil $\mathrm{D}_{\text {in }}$ and $\mathrm{D}_{\text {out }}$ & $70-280$ \\
$\mathrm{Rx}$ coil \# of turns & 16 \\
$\mathrm{Rx}$ coil inductances $(\mu \mathrm{H})$ & $65.6-65.4-66-64.9$ \\
$\mathrm{M}_{\mathrm{S}}(\mu \mathrm{H})$ & -10.7 \\
$\mathrm{C}_{\mathrm{Tx}}(\mathrm{nF})$ & 13.8 \\
$\mathrm{C}_{\mathrm{Rx}}(\mathrm{nF})$ & 21.1 \\
Airgap $(\mathrm{mm})$ & 30 \\
$\mathrm{f}_{0}$ & $150 \mathrm{kHz}$ \\
$\mathrm{f}$ & $155 \mathrm{kHz}$ \\
Ferrite shield & $\mathrm{I} 20 \mathrm{x} 2 \mathrm{~N} 48$ \\
\hline
\end{tabular}

\section{A. Fully-Aligned Case}

The inductance values of the full-aligned system are presented below in Table III. Current distributions for decoupled/fully-tuned, decoupled/detuned and coupled/fullytuned cases are presented in Fig. 12. Since the couplings between the Tx and Rx modules are very close, all Rx currents are nearly identical. However, since there exist small coupling and inductance differences current balancing effects of the proposed methods are observable.
TABLE III

EXPERIMENTAL INDUCTANCE VALUES

\begin{tabular}{l|ll|ll} 
& \multicolumn{2}{|l|}{ Experiment $(\mu H)$} & FEA $(\mu H)$ & \\
\hline & Fully-Aligned & Misaligned & Fully-Aligned & Misaligned \\
\hline $\mathrm{L}_{T x}$ & 83.5 & 83.5 & 80.95 & 80.95 \\
$\mathrm{~L}_{R x 1}$ & 65.5 & 65.6 & 63.7 & 63.7 \\
$\mathrm{~L}_{R x 2}$ & 65.4 & 65.4 & 63.7 & 63.7 \\
$\mathrm{M}_{1}$ & 13.9 & 12.3 & 15.86 & 13.71 \\
$\mathrm{M}_{2}$ & 14.5 & 15.2 & 15.86 & 16.9 \\
$\mathrm{M}_{s}$ & -10.7 & -10.7 & -9.1 & -9.1 \\
\hline
\end{tabular}

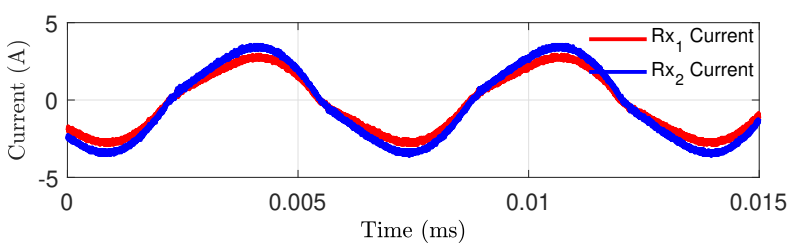

(a) $150 \mathrm{kHz}$ Decoupled Receivers $\left(\mathrm{I}_{\mathrm{Rx} 1}=1.9 \mathrm{~A}_{\mathrm{rms}}, \mathrm{I}_{\mathrm{Rx} 2}=2.4 \mathrm{~A}_{\mathrm{rms}}\right)$

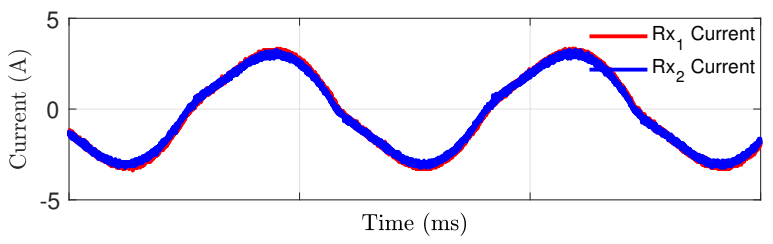

(b) $135 \mathrm{kHz}$ Decoupled Receivers $\left(\mathrm{I}_{\mathrm{Rx} 1}=2.2 \mathrm{~A}_{\mathrm{rms}}, \mathrm{I}_{\mathrm{Rx} 2}=2.1 \mathrm{~A}_{\mathrm{rms}}\right)$

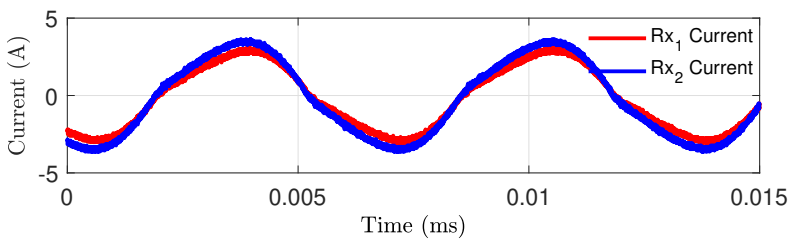

(c) $150 \mathrm{kHz}$ Cross-Coupled Receivers $\left(\mathrm{I}_{\mathrm{Rx} 1}=2.0 \mathrm{~A}_{\mathrm{rms}}, \mathrm{I}_{\mathrm{Rx} 2}=2.4 \mathrm{~A}_{\mathrm{rms}}\right)$

Fig. 12. Rx current waveforms for fully-aligned case and different current balancing methods.

\section{B. Misaligned Case}

The inductance values of the misaligned system are presented below in Table III. The current balancing effect comparisons are first done for cross-coupling effect and detuning and finally for the combined balancing methods. In Fig. 13-b, the effect of cross-coupling for fully-tuned Rx side is presented. In Fig. 13-c, the effect of detuning for no cross-coupling is presented. In Fig. 13-d, the combined current balancing effect of cross-coupled and detuned Rx modules is presented.

As seen from Fig. 13, the combined effect of the two proposed current balancing methods has a higher balancing ratio. This effect is also validated in a $2 \mathrm{Tx}-4 \mathrm{Rx}$ structure with a full circular geometry with rotating $\mathrm{Rx}$ coils. At this stage, it is important to discuss the effect of rotational speed on the current distribution. The $f_{0}$ was selected as $150 \mathrm{kHz}$ as given in Table I. For a rated rotational speed $\left(\mathrm{n}=1500 \mathrm{rpm}, \mathrm{f}_{\text {rotation }}=25 \mathrm{~Hz}\right)$, there exists 6000 gate switchings, which results in nearly 17 gate switching for a rotation of $1^{\circ}$. Since, the controller bandwidth of such a system is much higher, the system can be assumed stationary for any rotational position. In Fig. 14, the current waveforms in the $\mathrm{Rx}$ and $\mathrm{Rx}$ coils are presented for a complete rotation. At 


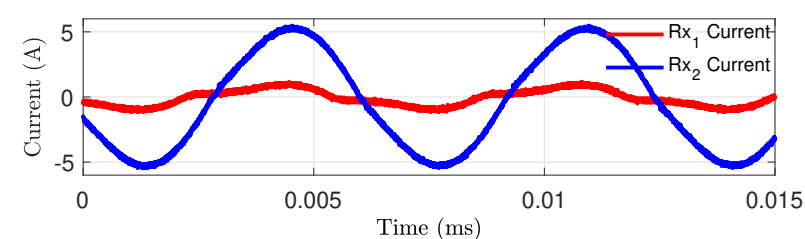

(a) $150 \mathrm{kHz}$ Decoupled Receivers $\left(\mathrm{I}_{\mathrm{Rx} 1}=0.6 \mathrm{~A}_{\mathrm{rms}}, \mathrm{I}_{\mathrm{Rx} 2}=3.7 \mathrm{~A}_{\mathrm{rms}}\right)$

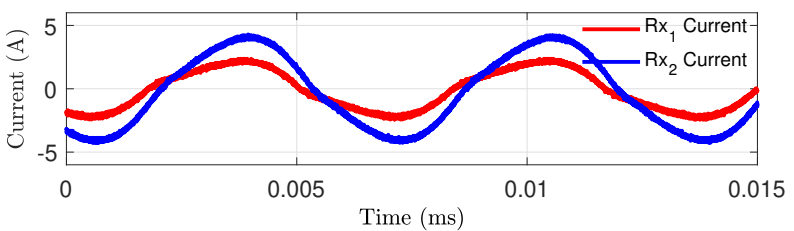

(b) $150 \mathrm{kHz}$ Cross-Coupled Receivers $\left(\mathrm{I}_{\mathrm{Rx} 1}=1.5 \mathrm{~A}_{\mathrm{rms}}, \mathrm{I}_{\mathrm{Rx} 2}=2.8 \mathrm{~A}_{\mathrm{rms}}\right)$

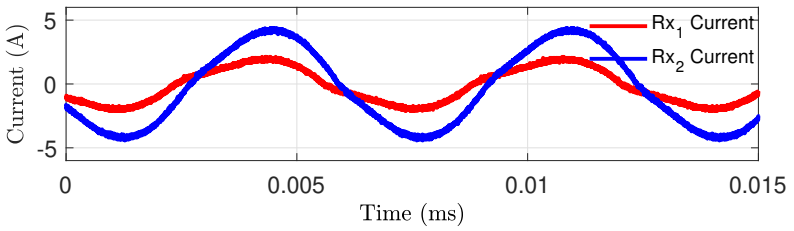

(c) $135 \mathrm{kHz}$ Decoupled Receivers $\left(\mathrm{I}_{\mathrm{Rx} 1}=1.3 \mathrm{~A}_{\mathrm{rms}}, \mathrm{I}_{\mathrm{Rx} 2}=2.9 \mathrm{~A}_{\mathrm{rms}}\right)$

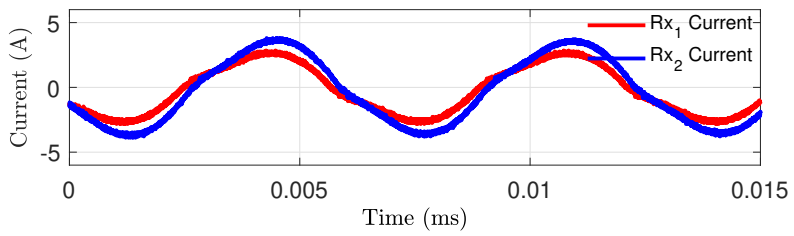

(d) $135 \mathrm{kHz}$ Cross-Coupled Receivers $\left(\mathrm{I}_{\mathrm{Rx} 1}=1.8 \mathrm{~A}_{\mathrm{rms}}, \mathrm{I}_{\mathrm{Rx} 2}=2.5 \mathrm{~A}_{\mathrm{rms}}\right)$

Fig. 13. Effects of cross-coupling and detuning on the Rx side current distribution for the missaligned case.

any given position, the Tx currents are nearly identical and constant. Therefore, it is possible to conclude that the reflected impedances to the Tx side are not only equal but also constant. For any given rotational angle, highly balanced $\mathrm{Rx}$ side module currents are achieved compared to Fig. 13-a.

\section{DISCUSSION}

In this section, firstly the experimental results will be discussed. Then the gain plots of the proposed methods will be presented. Let us define a current balance ratio (CBR) as in (44).

$$
\operatorname{CBR}(\%)=100\left(1-\frac{\left|\mathrm{I}_{\mathrm{Rx} 1}-\mathrm{I}_{\mathrm{Rx} 2}\right|}{\mathrm{I}_{\text {total }}}\right)
$$

When each Rx module currents are equal, CBR is $100 \%$. Experiment results are presented in Table IV and the effect of current balancing methods are included. As shown in Table IV, there is 55.8\% improvement in the current sharing compared to the de-coupled and fully-tuned case. The Rx side coil AC resistance was measured as $0.3 \Omega$. The total Rx side coil ohmic loss was also given in Table IV. Although the output power is kept constant for each experiment, the Rx side coil ohmic losses increases as the system becomes unbalanced due to an increased sum of $I_{R x, 1}^{2} R+I_{R x, 2}^{2} R+I_{R x, 3}^{2} R+I_{R x, 4}^{2} R$. The same is valid for Rx side rectifier diodes conduction losses.
Another critical issue is the variation of the overall system gain characteristics when cross-coupling and $\mathrm{Rx}$ side de-tuning methods are introduced. In Fig. 15, the gain characteristics for different current balancing methods are presented under fullyaligned position. The initial system design resonant frequency was selected as $150 \mathrm{kHz}$ as in Table I. As we introduce a negative cross-coupling, the peak of gain plots shifts to lower frequencies. The increase in the gain at the resonant frequency is expected as the Rx coils also induces an EMF component on each other, which further increases the output voltage as previously discussed in Fig. 6. De-tuning the Rx modules such that the resonant frequency is lower shifts the gain plot to higher frequencies. This is due to the reflected impedance of the Rx side to the Tx side. The reflected impedance can be expressed as in (45).

$Z_{r}=\frac{\omega^{2} M_{1}^{2}}{R_{e q u, 1}+j \omega L_{R x}+\frac{1}{j \omega C_{R x}}}+\frac{\omega^{2} M_{2}^{2}}{R_{e q u, 2}+j \omega L_{R x}+\frac{1}{j \omega C_{R x}}}$

Decreasing the Rx side resonant frequency may have the same effect as increasing the series capacitor. With a higher $\mathrm{Rx}$ side capacitance, a higher frequency is needed for the resonance. A similar statement can be made for the opposite case where the Rx side resonant frequency is adjusted higher than the resonant frequency of the Tx side. In this case, the resonant frequency decreases.

During the overall system design, a maximum allowable current unbalance of $50 \%$ was selected. Using the analytical model presented in the fourth section, resulted in either positive or negative cross-coupling with a magnitude of minimum of 0.15 . The addition of negative cross-coupling is achieved by an up-curvature IPT coil design as given in Fig. 1-a. By controlling area of the curvature of two Rx coil, the cross-coupling can be adjusted without a significant change in the self inductance. A similar geometry is also selected for Tx coils however, ferrite shielding was performed between the curvature of $\mathrm{Tx}$ coils in order to reduce the coupling between Tx modules. By eliminating the effect of the end windings, a much more uniform B-field was achieved in the airgap. A more detailed discusssion of IPT coil design is beyond the scope of this paper.

To further balance the currents, $135 \mathrm{kHz}$ is selected as $\mathrm{Rx}$ side resonant frequency while the Tx side is adjusted to $150 \mathrm{kHz}$. Compared to the initial design given in Fig. 15 (150 kHz Decoupled), the gain characteristics of the finalized design is nearly the same.

\section{SUMMARY}

In this paper, two new current balancing method for common DC-bus connected, modular, multi-Tx, and multi- Rx IPT system was presented. The current balancing was achieved during the design procedure and without any extra DCDC converters at the output. The analytical derivations are performed for the $1 \mathrm{Tx}-2 \mathrm{Rx}$ case using first harmonic approximation. The first method is the introduction of cross-coupling, which results in a $41 \%$ improvement in the current balancing. The second method is the intentional de-tuning of Rx side. 


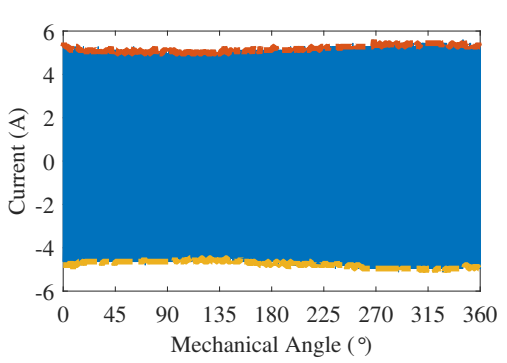

(a) Transmitter 1 .

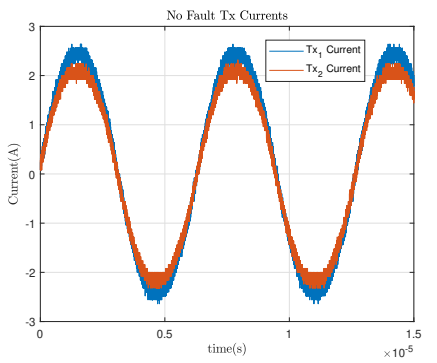

(b) Zoomed image of Tx current envelopes.

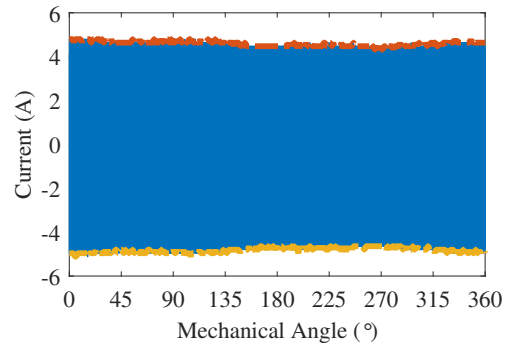

(c) Transmitter 2.

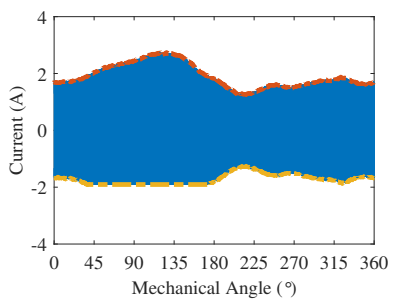

(d) Receiver 1.

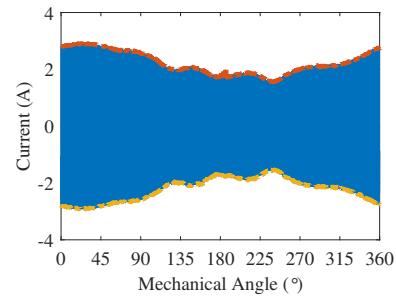

(e) Receiver 2 .

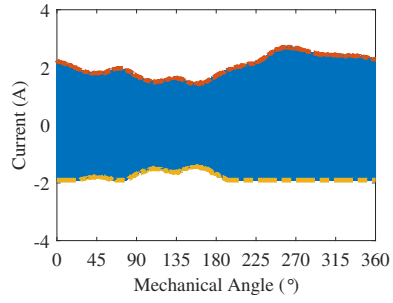

(f) Receiver 3 .

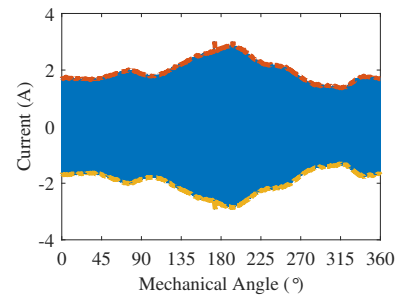

(g) Receiver 4.

Fig. 14. Tx and Rx current envelope waveforms for a complete revolution.

TABLE IV

CURRent Sharing for Missaligned Different Balancing Methods and Improvements

\begin{tabular}{lllll} 
& Decoupled Rx/Fully Tuned & Cross-Coupled Rx/Fully Tuned & Decoupled Rx/De-tuned & Cross-Coupled Rx/De-tuned \\
\hline Rx-1 Current & $0.6\left(A_{r m s}\right)$ & $1.5\left(A_{r m s}\right)$ & $1.3\left(A_{r m s}\right)$ & $1.8\left(A_{r m s}\right)$ \\
Rx-2 Current & $3.7\left(A_{r m s}\right)$ & $2.8\left(A_{r m s}\right)$ & $2.9\left(A_{r m s}\right)$ & $2.5\left(A_{r m s}\right)$ \\
Rx Copper Loss & $4.21(\mathrm{~W})$ & $3.03(\mathrm{~W})$ & $3.05(\mathrm{~W})$ & $2.85(\mathrm{~W})$ \\
CBR & $27.9(\%)$ & $69.7(\%)$ & $61.9(\%)$ & $83.7(\%)$ \\
Improvement & & $41.8(\%)$ & $34(\%)$ & $55.8(\%)$ \\
\hline
\end{tabular}

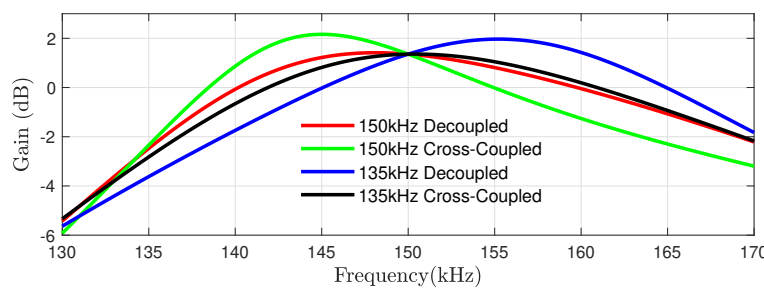

(a)

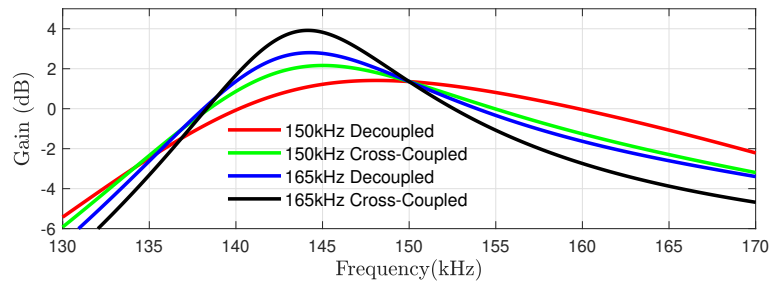

(b)

Fig. 15. $\frac{V_{\text {out }}}{V_{\text {in }}}$ gain plots for different current balancing methods.

By changing the resonant frequency, 34\% improvement was achieved. With the combination of these methods, the CBR was increased to $83.7 \%$. A similar improvement was also seen in the Rx side IPT coil ohmic losses, where a much more balanced current distribution resulted in a more efficient system.

\section{REFERENCES}

[1] G. A. Covic and J. T. Boys, "Modern trends in inductive power transfer for transportation applications," IEEE Journal of Emerging and Selected Topics in Power Electronics, vol. 1, no. 1, pp. 28-41, 2013.

[2] H. Hao, G. A. Covic, and J. T. Boys, "A parallel topology for inductive power transfer power supplies," IEEE Trans. Power Electron., vol. 29, no. 3, pp. 1140-1151, 2014.

[3] S. Y. R. Hui and W. W. C. Ho, "A new generation of universal contactless battery charging platform for portable consumer electronic equipment," IEEE Trans. Power Electron., vol. 20, no. 3, pp. 620-627, 2005.

[4] Y. Jang and M. M. Jovanović, "A contactless electrical energy transmission system for portable-telephone battery chargers," IEEE Trans. Ind. Electron., vol. 50, no. 3, pp. 520-527, 2003.

[5] O. Knecht, R. Bosshard, and J. W. Kolar, "High-Efficiency Transcutaneous Energy Transfer for Implantable Mechanical Heart Support Systems," IEEE Trans. Power Electron., vol. 30, no. 11, pp. 6221-6236, 2015.

[6] D. Patil, M. K. McDonough, J. M. Miller, B. Fahimi, and P. T. Balsara, "Wireless power transfer for vehicular applications: Overview and challenges," IEEE Trans. Transport. Electrific., vol. 4, no. 1, pp. 3-37, March 2018.

[7] M. Fu, H. Yin, M. Liu, Y. Wang, and C. Ma, "A $6.78 \mathrm{mhz}$ multiplereceiver wireless power transfer system with constant output voltage and optimum efficiency," IEEE Trans. Power Electron., vol. 33, no. 6, pp. $5330-5340,2018$

[8] V. B. Vu, V. T. Phan, M. Dahidah, and V. Pickert, "Multiple Output Inductive Charger for Electric Vehicles," IEEE Trans. Power Electron., vol. 34, no. 8, pp. 7350-7368, 2019.

[9] M. Q. Nguyen, Y. Chou, D. Plesa, S. Rao, and J. C. Chiao, "MultipleInputs and Multiple-Outputs Wireless Power Combining and Delivering Systems," IEEE Trans. Power Electron., vol. 30, no. 11, pp. 6254-6263, 2015.

[10] J. Pries, V. P. N. Galigekere, O. C. Onar, and G. J. Su, "A 50-kW ThreePhase Wireless Power Transfer System Using Bipolar Windings and 
Series Resonant Networks for Rotating Magnetic Fields," IEEE Trans. Power Electron., vol. 35, no. 5, pp. 4500-4517, 2020.

[11] Y. Song, U. K. Madawala, D. J. Thrimawithana, and M. Vilathgamuwa, "Three-phase bi-directional wireless ev charging system with high tolerance to pad misalignment," IET Power Electronics, vol. 12, no. 10, pp. 2697-2705, 2019.

[12] D. Ahn and S. Hong, "Effect of coupling between multiple transmitters or multiple receivers on wireless power transfer," IEEE Trans. Ind. Electron., vol. 60, no. 7, pp. 2602-2613, 2013.

[13] H. Zhou, J. Chen, Q. Deng, F. Chen, A. Zhu, W. Hu, and X. Gao, "Inputseries output-equivalent-parallel multi-inverter system for high-voltage and high-power wireless power transfer," IEEE Transactions on Power Electronics, vol. 36, no. 1, pp. 228-238, 2021.

[14] Z. Pantic, K. Lee, and S. M. Lukic, "Receivers for multifrequency wireless power transfer: Design for minimum interference," IEEE Journal of Emerging and Selected Topics in Power Electronics, vol. 3, no. 1, pp. 234-241, 2015.

[15] K. Colak, E. Asa, M. Bojarski, D. Czarkowski, and O. C. Onar, "A Novel Phase-Shift Control of Semibridgeless Active Rectifier for Wireless Power Transfer," IEEE Trans. Power Electron., vol. 30, no. 11, pp. 62886297, 2015.
[16] M. Fu, C. Ma, and X. Zhu, "A cascaded boost-buck converter for high-efficiency wireless power transfer systems," IEEE Transactions on Industrial Informatics, vol. 10, no. 3, pp. 1972-1980, 2014.

[17] G. Ke, Q. Chen, W. Gao, S. Wong, C. K. Tse, and Z. Zhang, "Research on ipt resonant converters with high misalignment tolerance using multicoil receiver set," IEEE Transactions on Power Electronics, vol. 35, no. 4, pp. 3697-3712, 2020.

[18] J. P. W. Chow, N. Chen, H. S. H. Chung, and L. L. H. Chan, "An investigation into the use of orthogonal winding in loosely coupled link for improving power transfer efficiency under coil misalignment," IEEE Transactions on Power Electronics, vol. 30, no. 10, pp. 5632-5649, 2015.

[19] H. Feng, T. Cai, S. Duan, X. Zhang, H. Hu, and J. Niu, "A dual-sidedetuned series-series compensated resonant converter for wide charging region in a wireless power transfer system," IEEE Trans. Ind. Electron., vol. 65, no. 3, pp. 2177-2188, 2018.

[20] Y. H. Sohn, B. H. Choi, E. S. Lee, G. C. Lim, G. Cho, and C. T. Rim, "General unified analyses of two-capacitor inductive power transfer systems: Equivalence of current-source ss and sp compensations," IEEE Transactions on Power Electronics, vol. 30, no. 11, pp. 6030-6045, 2015. 